\title{
Skyrmion Battery Effect via Inhomogeneous Magnetic Anisotropy
}

Xiawei Hao, ${ }^{1}$ Fengjun Zhuo, ${ }^{2}$ Aurélien Manchon, ${ }^{3,2}$ Xiaolin Wang, ${ }^{4} \mathrm{Hang} \mathrm{Li}^{1}{ }^{1, \text { a) }}$ and Zhenxiang Cheng ${ }^{4}$, b)

1) School of Physics and Electronics, Henan University, Kaifeng 475004, China

${ }^{2)}$ Physical Science and Engineering Division (PSE), King Abdullah University of Science and Technology (KAUST),

Thuwal 23955-6900, Saudi Arabia

${ }^{3)}$ Interdisciplinary Nanoscience Center of Marseille (CINaM), Aix-Marseille University, Marseille 13288,

France

${ }^{4)}$ Institute for Superconducting and Electronic Materials, Australian Institute of Innovative Materials,

University of Wollongong, Innovation Campus, Squires Way, North Wollongong, NSW 2500,

Australia

(Dated: 17 March 2021)

Magnetic skyrmions are considered as a promising candidate for the next-generation information processing technology. Being topologically robust, magnetic skyrmions are swirling spin textures that can be used in a broad range of applications from memory devices, logic circuits, to neuromorphic computing. In a magnetic medium lacking inversion symmetry, magnetic skyrmion arises as a result of the interplay between magnetic exchange interactions, Dzyaloshinskii-Moriya interaction, and magnetic anisotropy. Instrumental to the integrated skyrmion-based applications are the creation and manipulation of magnetic skyrmions at a designated location, absent any need of a magnetic field. In this paper, we propose a generic design strategy to achieve that and a model system to demonstrate its feasibility. By implementing in a disk-shaped thin film heterostructure an inhomogeneous perpendicular magnetic anisotropy, stable sub-100-nm size skyrmions can be generated without magnetic field. This structure can be etched out via, for example, focused ion beam microscope. Using micromagnetic simulation, we show that such heterostructure not only stabilizes the edge spins of the skyrmion, but also protects its rotation symmetry. Furthermore, we may switch the spin texture between skyrmionic and vortex-like ones by tuning the slope of perpendicular anisotropy using a bias voltage. When embedded into a magnetic conductor and under a spin polarized current, such heterostructure emits skyrmions continuously and may function as a skyrmion source. This unique phenomenon is dubbed as skyrmion battery effect. Our proposal may open a novel venue for the realization of all-electric skyrmion-based device.

\section{INTRODUCTION}

Magnetic skyrmion is a topologically nontrivial local spin texture $^{1}$ that results from competition among magnetic exchange, Dzyaloshinskii-Moriya interaction (DMI) and external field ${ }^{2,3}$. Skyrmion was first observed in MnGe - a helimagnet B20 crystal - using small angle neutron scattering ${ }^{4}$ and found later in metallic multilayers ${ }^{5}$, multiferroic magnets ${ }^{6}$, Heusler compounds ${ }^{7}$, perovskite heterostructures ${ }^{8}$, and twodimensional Van der Waals materials ${ }^{9,10}$. Creation and annihilation of skyrmions have been achieved by the application of magnetic field ${ }^{11,12}$, electrical field ${ }^{13}$, laser pulse ${ }^{14}$, and acoustic waves ${ }^{15,16}$. The magnetization profile of magnetic skyrmions can adopt several configurations that are topologically different: $\mathrm{Bloch}^{4}$, $\mathrm{Néel}^{5}$, anitskyrmion ${ }^{7}$, skyrmionium $^{17}$, meron ${ }^{18}$, bimeron ${ }^{19-21}$, bobbers ${ }^{22}$ and fractional skyrmion ${ }^{23}$. Despite its inhomogeneous magnetization, skyrmion exhibits particle-like motion that can be driven by a fairly low electric ${ }^{24}$ or thermal current ${ }^{25}$. In the past decade, significant effort has been made to detect, nucleate, stabilize, and transport skyrmions. Compared to conventional domain walls, skyrmions are not less sensitive to impurities and defects and allow much lower electric currents to be driven. It is for these reasons touted as a promising candidate for racetrack memory ${ }^{26}$, logic gate $^{27,28}$, probabilistic computing ${ }^{29}$, and neuromorphic devices ${ }^{30}$.

a)Electronic mail: hang.li@ vip.henu.edu.cn

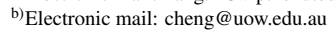

On the roadmap of skyrmion-based applications, creation and stabilization of skyrmions are of high priority ${ }^{2,3}$. In most two-dimensional systems that endow skyrmion, such as helimagnet films and heavy metal/ferromagnet bilayers, the ground state is usually non-skyrmionic ${ }^{4,5}$. A magnetic field alters the energy landscape of the system and allows the skyrmion formation to be energetically favorable, leading to metastable skyrmions or skyrmion lattice ${ }^{11,12}$. The pursuit of smaller yet more integrated nanostructures, however, craves for ingredients and mechanisms that can be used to manipulate skyrmions without cumbersome magnetic fields. Spin current is an outstanding candidate ${ }^{31-34}$. Sampaio et al. have demonstrated that spin current can nucleate an isolated skyrmion in a nanodisk and further drive it along a nanostrip ${ }^{35}$. Meanwhile, a system of confined geometry, when teamed up with magnetic anisotropies, provides a promising venue to realize fieldfree skyrmion. For example, the so-called target skyrmion is found stable in a FeGe nanodisk ${ }^{36}$. Engineering magnetic anisotropy even allows vortices to survive ${ }^{37}$. In another experiment ${ }^{38}$ performed in a confined $\mathrm{Co} / \mathrm{Ni} / \mathrm{Cu}$ multilayer, the perpendicular magnetic anisotropy balances the competition between DMI and exchange interaction, and modifies the energy profiles of metallic systems, leading to a stable skyrmion.

In a nano-sized device, to generate skyrmions at a designated location is a highly desirable feature yet heretofore a major challenge. In one proposal that employs spin waves, skyrmion was generated in the designated location by tuning the microwave field ${ }^{39}$. Another viable approach is to preserve the rotational symmetry of skyrmion by confining the 
spin-spiraling structure in a nanodisk $\mathrm{k}^{35,36}$ on which skyrmions can nucleate in the presence of a magnetic field or local spin current ${ }^{40}$. In these setups, only the central or edge magnetization are anchored by magnetic field or local spin current, due to the fact that the local energy density of the skyrmion decays from the center towards the edge ${ }^{41}$, i.e., a stable skyrmion favors a spatially inhomogeneous energy density. Skyrmions can also be generated in the vicinity of crystal grain ${ }^{42}$, defect ${ }^{43-46}$ and a constrained boundary by a current $^{47,48}$. In this case, local magnetization that is tilted inhomogeneously evolves into a spin spiral and further into a vortex in the constrained region. In the vicinity of the grain, the magnetic anisotropy (hence the spin helical axes) undergoes local modification, which points to the possibility to use inhomogeneous magnetic anisotropy to create desired spin textures. For example, Luo et al. employed an inhomogeneous magnetic anisotropy to create chiral domain walls ${ }^{49}$ In this case, an in-plane (out-of-plane) magnetic anisotropy is generated in the left (right) part of a rectangle. Near the interface, a chiral-dependent domain wall is created and stabilized even in the absence of magnetic field. Inhomogeneity in magnetic anisotropy also exerts impact on the motion of skyrmions. In the presence of a carefully patterned anisotropy gradient $t^{50-52}$, a skyrmion driven by a voltage pulse can travel more than $500 \mathrm{~nm}$. An interlayer coupling in a synthetic antiferromagnetic bilayer structure with an anisotropy gradient can further enhance the skyrmion velocity ${ }^{53}$.

In this paper, we propose a design pattern and a model system to generate and stablise skyrmions using inhomogeneous magnetic anisotropy in FeGe-based ultrathin films. Schematic views of the model system are shown in Fig. 1. Key ingredient to this setup is the material variation along the radial direction (of the disk) that is realized by a capping layer deposited a the center (region I) and is surrounded by a circular defective region (region II). The defective region can be etched using a side mask ${ }^{49}$ or a focused ion beam microscope ${ }^{46}$. The capping layer is expected to enhance the perpendicular magnetic anisotropy (PMA). We show that, owing to this particular design, anisotropy energy varies inhomogeneously along the radius, which allows (1) skyrmions and to nucleate in a FeGe nanodisk, and (2) a possible transition from skyrmion to vortex to occur by tuning the PMA via, for example, an applied bias voltage. More interestingly, when embedded into a magnetic conductor that endows skyrmions, this model system may emit skyrmions continuously under the stimulation of a spin polarized current, a phenomenon we shall call skyrmion battery effect.

We organize this paper as follows. In Sec.II, we propose a model system to integrate inhomogeneous perpendicular anisotropy and outline the simulation methods. Section III is dedicated to a comparative study that includes a case in which magnetic anisotropy is absent. A phase diagram is made to highlight the competition between exchange and DMI in generating different magnetization configurations. Motivated by the results in Sec.III, we propose two configurations - central well and central barrier - in Sec.IV to realize inhomogeneous perpendicular anisotropy. For each configuration, the underlying model system is studied as an individual device. In Sec.V, (a)

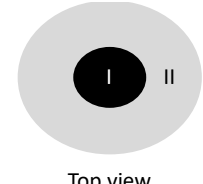

(b)

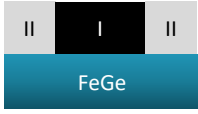

Side view

(c)

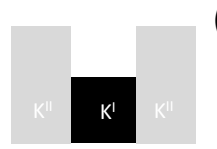

(d)

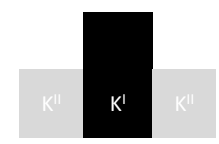

FIG. 1. A schematic view of the model system. The system is diskshaped heterostructure made of ultrathin bilayer films. A capping layer that is partitioned into region I (black) and region II (grey) is deposited on a ferromagnetic FeGe film. The perpendicular magnetic anisotropy in region I is different from that in region II, which induces in the FeGe layer an inhomogeneous magnetic anisotropy. (a) Top and (b) side views of the nanodisk. Panels (c) and (d) show the spatial profiles of the perpendicular magnetic anisotropy on the cross section. Panel (c) refers to the case where the anisotropy constant $K_{u}^{(\mathrm{I})}$ in the central region (I) is larger than $K_{u}^{(\mathrm{II})}$ in region II. Panel (d) refers to the opposite case.

these devices are further embedded as elements into a larger magnetic nanostrip to investigate current-induced effects. We found a critical current density beyond which skyrmion develops a current-induced motion; a lower current density only powers local deformation of skyrmion. We discuss skyrmion battery effect in this section and one of its potential applications in Sec.VI.

\section{MODEL SYSTEM AND SIMULATION METHODS}

Consider a model system sketched in Fig. 1. External magnetic field is absent. This disk-shaped device is a heterostructure that consists of a capping layer - partitioned into region I and II - deposited on top of a FeGe ultrathin film (blue) which endows an perpendicular magnetic anisotropy ${ }^{54}$. Nucleation of skyrmion is expected to occur in the FeGe layer. This particular design modulates - via exchange couplings induced by different materials - the magnitude of the anisotropy and is thus creating along the radial direction an inhomogeneous PMA in the FeGe film, see Figs. 1(c) and (d). Material wise, an iron layer in region I induces a ferromagnetic coupling with the adjacent FeGe film ${ }^{55,56}$, whereas an EuS film ${ }^{57}$ favours an antiferromagnetic one. On the other hand, metallic oxides, such as $\mathrm{MgO}^{58}$ or $\mathrm{Ir} / \mathrm{MgO}^{59}$, on the capping layer may further enhance the perpendicular anisotropy via orbital hybridization between oxygen and iron atoms. An inhomogeneously distributed magnetic anisotropy has been realized by Luo et. al. ${ }^{49}$ and the orientation and magnitude of the perpendicular anisotropy can be tuned by a gate voltage ${ }^{60}$. To reveal the role of an inhomogeneous perpendicular magnetic anisotropy in the stabilization of skyrmions, we conduct a 
comparative study using two setups: The perpendicular magnetic anisotropy is homogeneous in one setup and inhomogeneous in the other.

Within the framework of micromagnetism or micromagnetic simulations, the magnetization dynamics in the presence of a spin current is governed by the augmented LandauLifshitz-Gilbert (LLG) equation

$$
\frac{d \vec{M}}{d t}=-\gamma \vec{M} \times \vec{H}_{\mathrm{eff}}+\frac{\alpha}{M_{s}} \overrightarrow{\boldsymbol{M}} \times \frac{d \vec{M}}{d t}-(\overrightarrow{\boldsymbol{u}} \cdot \nabla) \vec{M},
$$

where $\vec{M}$ is the unit vector of the local magnetization, $\gamma$ is the gyromagnetic ratio, $\alpha$ is the Gilbert damping constant, and $M_{s}$ is the saturation magnetization. In this dynamic equation, the effective field applied on the unit cell is obtained by $\overrightarrow{\boldsymbol{H}}_{\text {eff }}=-\partial \mathscr{E} /\left(\mu_{0} \partial \overrightarrow{\boldsymbol{M}}\right)$, given $\mu_{0}$ the permeability of free space. The total magnetic energy $\mathscr{E}$ incorporates the exchange coupling, anisotropy, magnetostatic energy, and DMI. In the present model, the effective field reads

$$
\begin{aligned}
\overrightarrow{\boldsymbol{H}}_{\mathrm{eff}}= & \frac{2 A_{\mathrm{ex}}}{\mu_{0} M_{s}^{2}} \nabla^{2} \overrightarrow{\boldsymbol{M}}+\frac{2 K_{u}^{(i)}}{\mu_{0} M_{s}^{2}}(\overrightarrow{\boldsymbol{M}} \cdot \hat{\boldsymbol{z}}) \hat{\boldsymbol{z}} \\
& -\frac{2 D}{\mu_{0} M_{s}^{2}}(\nabla \times \vec{M})+\mathscr{K}_{i j} \vec{M}
\end{aligned}
$$

where $A_{\text {ex }}$ is the exchange stiffness ${ }^{61}, D$ is the DMI constant ${ }^{1}$, and $\mathscr{K}_{i j}$ is the demagnetizing tensor ${ }^{62-64}$. Anisotropy constant $K_{u}^{(i)}$ of a magnetic cell has value $K_{u}^{(\mathrm{I})}\left(K_{u}^{(\mathrm{II})}\right)$ in region I (II). The last term on the right-hand side of the LLG equation (1) is the adiabatic spin transfer torque induced by a spin current that is (spin) polarized to the $\mathrm{z}$ axis ${ }^{65}$. Vector $\boldsymbol{u}$ is along the electron flow and its magnitude $u=j P \mu_{B} /\left(e M_{s}\right)$ is proportional to the electric current density $j$ and its spin polarization $P$, given $\mu_{B}$ Bohr magneton and $e$ the absolute value of elementary charge. In the simulation, the variation of DMI coupling in the radial direction due to material variation is neglected. This is further justified by a sufficiently strong anisotropy at the edge or center, which is usually valid in experimental conditions.

All simulations are performed using the MuMax3 package ${ }^{62}$. The size of the unit cell is $2.5 \mathrm{~nm} \times 2.5 \mathrm{~nm} \times$ $2.5 \mathrm{~nm}$; any dimension is shorter than the exchange length $l_{\mathrm{ex}}=\sqrt{2 A_{\mathrm{ex}} / \mu_{0} M_{s}^{2}} \sim 9.7 \mathrm{~nm}$. In all simulations, unless otherwise specified, we adopt the following paraemter values: $A_{\mathrm{ex}}=8.78 \mathrm{pJ} \mathrm{m}^{-1}, M_{s}=3.84 \times 10^{5} \mathrm{~A} \mathrm{~m}^{-1}, D=1.58 \times 10^{-3}$ $\mathrm{J} \mathrm{m}^{-266}$. The thickness of the device is $10 \mathrm{~nm}$. The diameter of the nanodisk is set to $100 \mathrm{~nm}$ and the width of the ringshaped region II in Fig. 2(a) is $70 \mathrm{~nm}$. In the discussions below, unless otherwise specified, we omitted for the brevity of the notation the unit $\left(\mathrm{MJ} \mathrm{m}^{-3}\right)$ associated with the anisotropy constants $K_{u}^{(\mathrm{I})}$ and $K_{u}^{(\mathrm{II})}$, for example, $K_{u}^{(\mathrm{I})}=0.3$ is understood as $K_{u}^{(\mathrm{I})}=0.3 \mathrm{MJ} \mathrm{m}^{-3}$.

For the initial magnetization configuration, or simply initial state, we consider four configurations, namely, Bloch skyrmion $(B)$, ferromagnetic $(\mathrm{F})$, helical $(\mathrm{H})$, and random orientation $(\mathrm{R})$ and assume that the magnetization profile for each layer is identical. At zero temperature, the initial state evolves according to the LLG equation. The relaxation time is set to $200 \mathrm{~ns}$. Figure 2 clearly shows that, in our simulations, the final magnetization configuration (or final state) is dependent on the choice of the initial state.

\section{ABSENCE OF ANISOTROPY}

The role of perpendicular anisotropy in facilitating skyrmion generation is the central topic this paper addresses. We first provide in Fig. 2 a magnetic phase diagram - spatial profile of the $z$ component of the magnetization - of a nanodisk in the absence of perpendicular magnetic anisotropy and polarized current. From a given initial state, the system evolves into a final state, which is governed by LLG equation (1). For a disk with a small diameter $(\leq 90 \mathrm{~nm})$, the final states are degenerate in energy. In Fig.2, we attach a green triangle to label a final state that has the lowest energy under the same diameter (column). In a finite-sized system, vortex-like state is energetically favored in the presence of a sufficiently large DM interaction ${ }^{67,68}$, which is especially so in a disk that has a small diameter: for disks of diameter ranging from 60 to $110 \mathrm{~nm}$, vortex-like state always has the lowest energy. Meanwhile, the presence of vortex-like state is independent of the device's in-plane geometry - square, triangle or circle ${ }^{69}$.

Important features of the system emerge from phase diagram. At the edge of the disk, spins deviate from the perpendicular direction, reducing energy and stabilizing the vortex structure. On the other hand, as the diameter of the disk is commensurate with the period of spin helix, spins at the edge prefer the perpendicular direction due to the interplay between DMI and exchange interaction. As a result of these two competing effects, the final stable state is sensitive to the size of the disk.

\section{PERPENDICULAR MAGNETIC ANISOTROPY}

Stability analysis in the last section suggests that a perpendicular magnetic anisotropy may mediate the competition between the DMI and exchange interaction ${ }^{2}$. A homogeneously distributed perpendicular anisotropy raises the threshold value of $D$ - which is proportional to anisotropy constant $K_{u}$ - required to generate a vortex ${ }^{71}$. In Fig.3, we show the final stable states versus $K_{u}^{(\mathrm{I})}$ and $K_{u}^{(\mathrm{II})}$. The case of homogeneous magnetic anisotropy $\left(K_{u}^{(\mathrm{I})}=K_{u}^{(\mathrm{II})}\right)$ is represented by the cells on the diagonal. A weak and homogeneous anisotropy $\left(K_{u}^{(\mathrm{I})}=\right.$ $\left.K_{u}^{(\mathrm{II})}<0.3\right)$ renders the final stable state helical. A homogeneous yet sufficiently large anisotropy, say $K_{u}^{(\mathrm{I})}=K_{u}^{(\mathrm{II})}>0.3$, suppresses the spin spiral and the final stable state becomes ferromagnetic, as the lower right corner of Fig. 3 shows.

The most important feature shown in Fig.3 is that the difference between $K_{u}^{(\mathrm{I})}$ and $K_{u}^{(\mathrm{II})}$ creates non-ferromagnetic final states. When $K_{u}^{(\mathrm{II})} \geq 2 K_{u}^{(\mathrm{I})}$, shown on the top right corner of Fig.3, the final state becomes skyrmionic. On the opposite limit $K_{u}^{(\mathrm{I})} \geq 3 K_{u}^{(\mathrm{II})}$, the lower left corner of Fig.3, the final state is dominated by vortices. To understand the mechanism that 


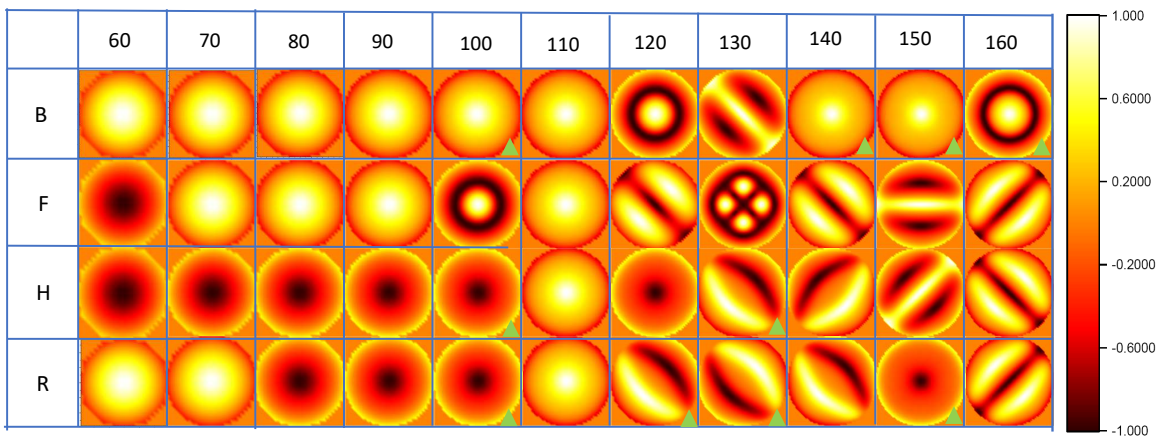

FIG. 2. The spatial profile of the $z$-component of the final magnetisation configuration in a nanodisk. Each cell represents the final configuration on a nanodisk with a given diameter (horizontal axis) and initial state (vertical axis). The initial state is prepared as one of the four options: Bloch-type skyrmion (B), ferromagnetic state $(\mathrm{F})$, helix $(\mathrm{H})$, and random $(\mathrm{R})$. The central spins of Bloch skyrmion are spin down and the magnetization of ferromagnetic state is spin up at $t=0$. The parameters are $A_{\mathrm{ex}}=8.78 \mathrm{pJ} \mathrm{m}^{-1}, D=1.58 \mathrm{~mJ} \mathrm{~m}^{-2}$ and $K_{u}=0$.

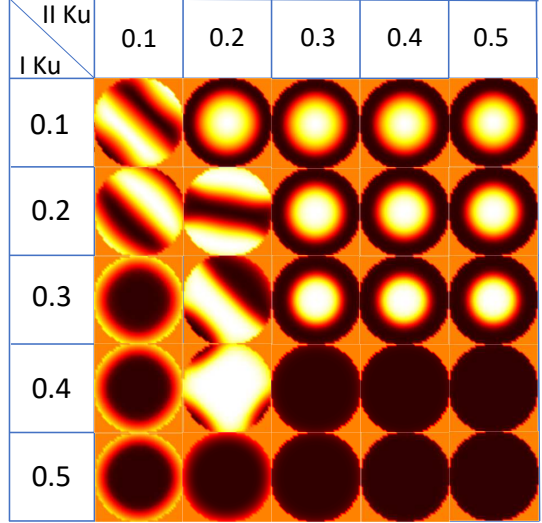

FIG. 3. Effect of perpendicular anisotropy. The spatial profile of the $z$-component of the magnetization on a nanodisk. Each cell represents the stable final state. Horizontal axis is the value of the anisotropy constant $K_{u}^{(\mathrm{II})}$ in region II; the vertical axis is $K_{u}^{(\mathrm{I})}$ in region I. The diameter of disk is $100 \mathrm{~nm}$ and others parameters remain the same as in Fig.2.

enables a step-like perpendicular magnetic anisotropy to generate and stabilize skyrmions, we must turn to the energies of the final state.

\section{A. Central well $\left(K_{u}^{(\text {I) }}<K_{u}^{(\text {(I) })}\right.$}

Figure 1(c) corresponds to this case. The energy of different final state as a function of $D$ is plotted in Fig. 4(a) for various $K_{u}^{(\mathrm{II})}$ and a constant $K_{u}^{(\mathrm{I})}=0.1$. (Only the magnetization configurations of the final stable states with the lowest energy are shown.) When $K_{u}^{(\mathrm{I})}=K_{u}^{(\mathrm{II})}=0.1$ (red line), the total energy decreases with $D$. The ferromagnetic state has the lowest energy as $D<1.0 \mathrm{~mJ} \mathrm{~m}^{-2}$, see Fig. 4(a), which agrees with an earlier report ${ }^{35}$. As $D$ increases, a helical state with a period that is proportional to $A_{\mathrm{ex}} / D$ becomes the stable state and it remains energetically more favorable (than skyrmionic state) even at greater $D$, for a greater $K_{u}$ means a higher threshold value of $D^{71}$. However, when $K_{u}^{(\mathrm{I})} \neq K_{u}^{(\mathrm{II})}$ (other colors), the magnetization configuration is skyrmionic for $D=1.0$ or $1.5 \mathrm{~mJ} \mathrm{~m}^{-2}$. As $K_{u}^{(\mathrm{II})}$ increases above 0.3 , the helical states are suppressed and final state is either skyrmion or target skyrmion.

To understand the role of PMA, we plot and compare the local energy density without and with a step-like perpendicular magnetic anisotropy in an instantaneous skyrmion (Fig. 4(b)) and a stable skyrmion (Fig. 4(c)). The local energy density in Fig. 4(b) varies smoothly from the edge to the center. The energy difference between the center and the edge is small and the effect of the energy barrier vanishes. In Fig. 4(c), the locally enhanced perpendicular magnetic anisotropy acts effectively as a potential well, which suppresses DMI at the edge and retains the rotational symmetry of the skyrmionic spin texture. The skyrmionic state is hence stable.

The final stable state is determined by the competition between the anisotropy and exchange interaction - both DMI and ferromagnetic exchange. To show this, we plot in Fig. 4(d) each component that contributes to the total energy density as a function of $D$ at various $K_{u}^{(I I)}$. From the left to right panels, the increase in relative dominance of anisotropy energy over other components - exchange and stray field - is 

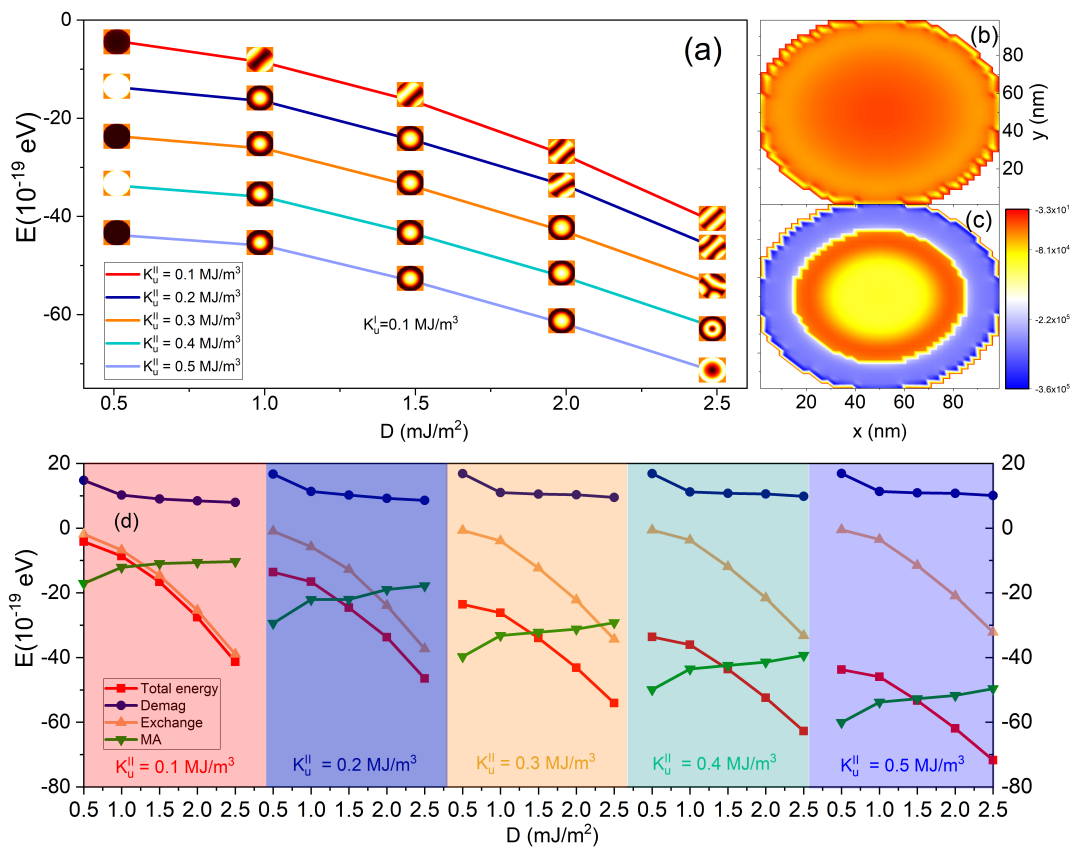

FIG. 4. (a) The total energy as a function of the DMI strength for different perpendicular magnetic anisotropy in region I and in region II. The perpendicular magnetic anisotropy in region I is smaller than (or equal to) it in region II. The local energy density of a skyrmion in a disk without (b) and with (c) a gradient perpendicular magnetic anisotropy. (d) The different components of total energy as function of varied PMA. The inset figure at each point in (a) represents the stable final state.

strongly correlated with the fact that a skyrmionic state is the final stable state.

\section{B. Central barrier $\left(K_{u}^{(1)}>K_{u}^{(11)}\right)$}

This case corresponds to Fig.1(d) where the anisotropy in the central region (I) is greater than that in the outer ring (II). In Fig. 5(a), the $K_{u}^{(\mathrm{I})}$ varies from $0.1 \sim 0.5 \mathrm{MJ} \mathrm{m}^{-3}$ and $K_{u}^{\text {(II) }}=0.1$. Similar to the central well, a weak DM interaction $\left(D<1.0 \mathrm{~mJ} \mathrm{~m}^{-2}\right)$ always leads the system into a ferromagnetic final state, as both the exchange and perpendicular anisotropy favor a uniform spin alignment. As $D$ increases $\left(D=1.0\right.$ or $\left.1.5 \mathrm{~mJ} \mathrm{~m}^{-2}\right)$, the final state is a vortex when $K_{u}^{(\mathrm{I})}$ is above 0.2 . This final state can be excited by a spin current injected into the core of nanodisk, too ${ }^{35}$. A spin-polarized current upon injection brings the central area into a polarized state, which plays - in a time window that is determined by the spin relaxation time - the role of an effective perpendicular anisotropy. In metallic multilayers such as $\mathrm{Co} / \mathrm{Pt}$, the period of the spin helix is about $50 \mathrm{~nm}$ and the role of DMI at the edge is weaker than that in FeGe nanodisk, hence it is more prac- tical to generate a skyrmionic state via spin current injection. A similar approach applies to a $\mathrm{FeGe}$ nanodisk. When $D$ rises above 2.0, the vortex evolves into a multi-domain or helical state to lower the global energy. Local energy density profile of an instantaneous skyrmion and a stable vortex is shown in Fig. 5(b) and (c), respectively. Opposite to its function in the central well configuration, the anisotropy in the central region now acts as a potential barrier and it lowers the topological charge of the magnetization configuration.

The two cases we have analyzed suggest a new possibility. If the perpendicular anisotropy can be tuned externally to be inhomogeneous, we may achieve the desired magnetization configurations. Recent experiment has shown that the magnitude of perpendicular magnetic anisotropy can indeed be tuned by a bias voltage, owing to the variation in the relative occupation of $3 \mathrm{~d}$ orbitals of $\mathrm{Fe}$ atoms which are adjacent to the $\mathrm{MgO}$ layer $^{58}$. This also makes possible a transition from, for example, a skyrmion to a vortex, or the reverse. Such transition is accompanied by a change in the topological charge and conductivity. Such feature - switching between two topologically nonequivalent states - can be used as an onsite binary element in spintronic devices. The repulsive nature 

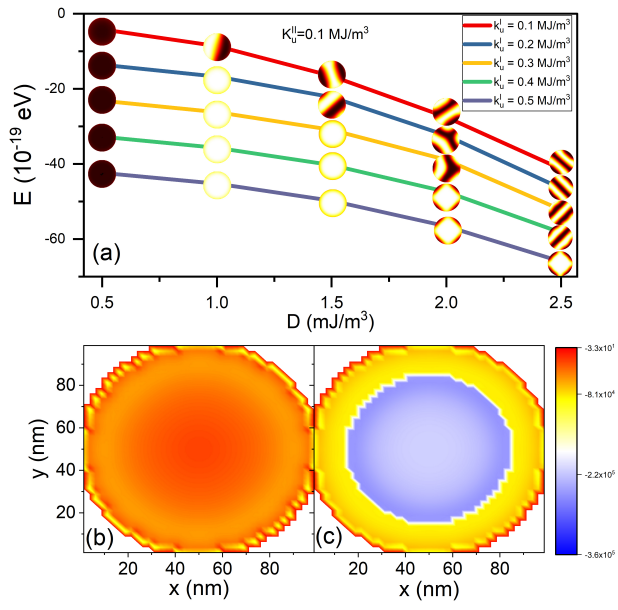

FIG. 5. (a) The total energy as a function of the DMI strength for different perpendicular magnetic anisotropy, where the perpendicula magnetic anisotropy in region I is larger or equal to it in region II. The local energy density of a skyrmion in a disk without (b) and with (c) a gradient perpendicular magnetic anisotropy. The inset figure at each point in (a) represents the stable final state.

of the barrier-shaped inhomogeneity can be used to correct the skyrmion trajectory, should it be implemented in a larger circuit where skyrmion flows.

\section{Thickness dependence}

In a two-dimensional film, both experiments and theories have shown that the range of magnetic field in which skyrmion is the ground state depends on the thickness. There exists a threshold value in thickness above which skyrmion becomes metastable. With finite thickness, the in-plane spin components on the top layer are rotated by a small angle relative to the ones on the bottom, dubbed as the chiral surface twist effect $^{22}$. This is due to the spin spiral structure along the vertical direction and it has lower energy than simply stacking, layer by layer, the skyrmions without interlayer twisting. We focus on an ultrathin film with a thickness below $25 \mathrm{~nm}$. Other effects such as chiral bobber is beyond the scope of this paper.

In Fig. 6, the spatial profile of the $z$-component of the magnetization is plotted for films with different thickness. When the DMI is weak, the spin at the edge is pinned to the $z$ direction and the profile is close to a cosine function, see Fig. 6(a) and (b). Increase in thickness only causes negligible deviation from cosine. With a large $D\left(2.5 \mathrm{~mJ} \mathrm{~m}^{-2}\right)$, the spatial profile - the spatial modulation - becomes largely thickness dependent, see Fig. 6(c). Change in thickness may cause the magnetization configuration to vary from skyrmionium to skyrmion. A strong DMI means a short helical period
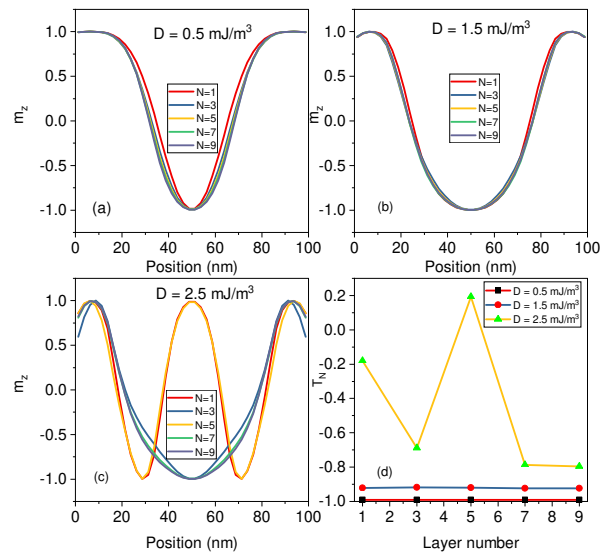

FIG. 6. Spatial profile of the magnetization ( $\mathrm{z}$ component) across the core of an skyrmion in a disk for the different thicknesses of a nanodisk for (a) $D=0.5 \mathrm{~mJ} \mathrm{~m}^{3}$, (b) $D=1.5 \mathrm{~mJ} \mathrm{~m}^{3}$ and (c) $D=2.5$ $\mathrm{mJ} \mathrm{m}^{3}$. (d) The topological number as a function of the thicknesses of nanodisk for the different DMI. N denotes the number of FeGe layer and $N=1$ is the single layer case.

and hence more periods the disk can accommodate. The thickness dependence of topological number is shown in Fig. 6(d). This indicates that the exchange coupling among the different layers along the $\mathrm{z}$ axis actually helps stabilize the skyrmionic state. We note that the imperfect topological number appearing in Fig. 6(d) is a result of the particular spin boundary condition induced by the finite size of the nanodisk.

\section{SKYRMION BATTERY EFFECT}

In this section, we embed into a larger conductor the element discussed in the previous section and study the motion of a skyrmion driven by a spin current ${ }^{24,70}$. Consider a nanostrip made of FeGe thin film, see Fig. 7(b). The lateral dimension of the nanostrip used in the simulations is $900 \mathrm{~nm} \times$ $500 \mathrm{~nm}$; the thickness of the film is $7.5 \mathrm{~nm}$. On the nanostrip, a disk-shaped area (referred to as defective area) with a lower perpendicular magnetic anisotropy is embedded to generate skyrmion, implementing the central well type configuration described in Sec.IV A. The nanostrip is prepared in a ferromagnetic state with a skyrmion initiated at the defective area. However, it appears that the final state of the nanostrip does not depend on its initial spin configuration. A spin current with spin polarized along $z$ is injected from the left edge. Under a low current, about $1.8 \sim 2.6 \times 10^{10} \mathrm{~A} \mathrm{~cm}^{-2}$, the skyrmion remains static and the most stable final state remains skyrmionic. In this limit, perturbation caused by the injected spin current is quickly dissipated via damping. As the current density increases, the skyrmion deforms into a vortex as a result of the rising magnus force induced by the current, 

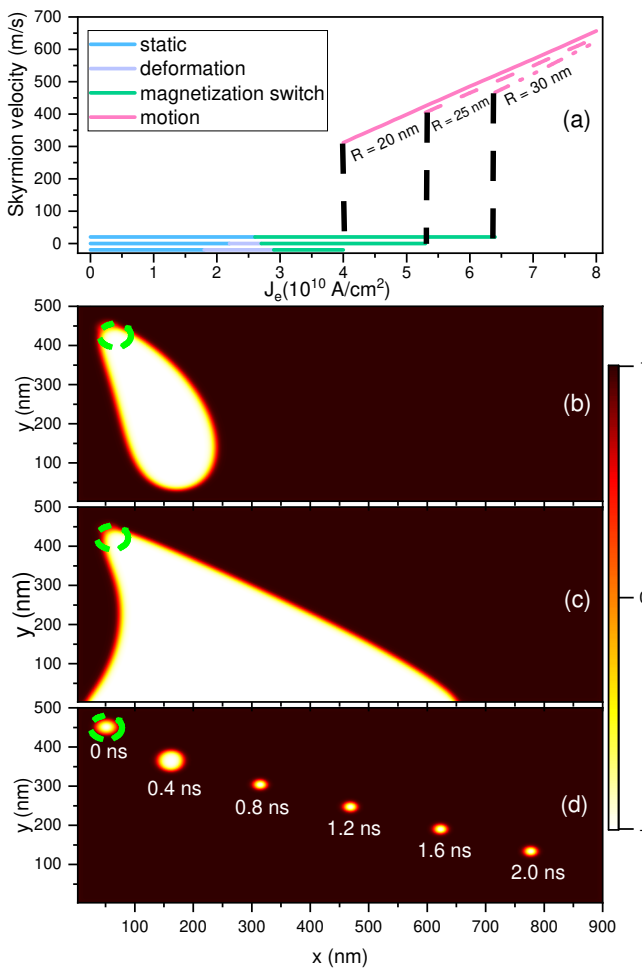

FIG. 7. Skyrmion creation and motion in a rectangle nanostrip under a polarized spin current. The dimension of the nanostrip is 900 $\mathrm{nm} \times 500 \mathrm{~nm}$. The skyrmion is generated in a disk-shape area located at the upper left corner of the rectangle. This area has a lower $K_{u} \sim 0.1$ than the rest of the nanostrip where $K_{u} \sim 0.3$. (a) Skyrmion velocity as a function of spin current density for various diameters of the disk, $40 \mathrm{~nm}$ (solid pink), $50 \mathrm{~nm}$ (dashed pink), and $60 \mathrm{~nm}$ (dash-dotted pink). (b) Profile of the $z$-component of the magnetization of the deformed vortex. (c) Magnetization switching due to the expansion of the deformed skyrmion. (d) Snapshot of skyrmion motion at different moments. Thickness of the FeGe strip is $7.5 \mathrm{~nm}$ and $D=1.0 \mathrm{~J} \mathrm{~m}^{-3}$. The nanostrip is prepared in a ferromagnetic initial state with a skyrmion at the defective area. The spin polarization of the current is along $z$. The green circle refers to the position of defect in the nanostrip.

see Fig. 7(b). In the simulation, the current density required to deform a skyrmion (purple line) depends on the radius of the embedded disk. Under a constant current density, the area of vortex is proportional to the diameter of the disk-shaped area Further increase in current results in a magnetization switching in a large area, see Fig. 7(c).

What is more interesting is the existence of a critical current above which the initiated skyrmion develops motion rather than deformation - from the upper left corner to lower right one. To provide an order-of-magnitude estimation to the threshold value, we use a formula derived for a system with homogeneous anisotropy ${ }^{71-73}$

$$
j_{\mathrm{th}} \approx \frac{2 \pi e M_{s} t_{n} v_{\mathrm{sk}} \sqrt{G^{2} \alpha^{2}+Q^{2}} \sqrt{2\left(1-D / D_{c}\right)}}{\pi \hbar \gamma_{0} P \sqrt{A_{e x} / K_{u}}}
$$

where the $t_{n}$ is the thickness of ferromagnetic layer, $G$ is the dissipative force constant, $Q$ is the skyrmion number, $D_{c}$ is the threshold value of $\mathrm{D}$ to create the skyrmion and $v_{s k}$ is skyrmion velocity. Consider for example a skyrmion with the radius $R_{s k}=20 \mathrm{~nm}$ (solid pink line) in Fig. 7(a). Equation (3) gives a threshold current $\sim 2 \times 10^{10} \mathrm{~A} \mathrm{~cm}^{-2}$, which agrees with the numerical result. The motion of the skyrmion, shown in Fig. 7(d), is accompanied by a breathing mode with a radius stabilized at about $30 \mathrm{~nm}$ and enjoys a lifetime that is long enough to reach the opposite edge. Figure 7(a) also shows the threshold value of current density depends on the diameter of the defective region, a feature that is of practical importance in device design. Due to its lower energy, the defective region "attracts" skyrmions moving nearby and this property has potential application in correcting the skyrmion trajectory. Note that the skyrmion can be driven by spin polarized current and travel $1800 \mathrm{~nm}$ in a larger system with the size $2000 \mathrm{~nm} \times$ $1250 \mathrm{~nm}$ (not shown in this paper).

A rather unique phenomenon occurs in this setup. Upon the injection of a spin current - polarized along $z$ and flowing along the $x$ direction - skyrmions are generated continuously in and pushed out of the defective area, as Fig. 8(a) shows. This is a natural consequence of inhomogeneous anisotropy mediated skyrmion generation and its current-driven motion. The underlying mechanisms are detailed in the previous sections.

We provide here a simple but intuitive picture. A moderate spin current pushes the skyrmion out of the potential well of the disk-shaped defective region, and what is left in the disk is a ferromagnetic state. However, the spin current is polarized along the $z$ direction and with a moderate density its net effect on the spin dynamics on the disk is similar to the field due to a perpendicular magnetic anisotropy. As long as the energy on the disk is dominated by the exchanges and anisotropy, a ferromagnetic state is not stable, see Fig. 3 and discussion in Sec.IV, and skyrmion nucleates in the defective region. This skyrmion is subsequently pushed out of the disk and the above cycle starts over. This continuous generation of skyrmions is dubbed as skyrmion battery effect.

The transition from an unstable ferromagnetic state to a skyrmion comes across an intermediate metastable state, a droplet state. Figure 9 consists of snapshots of this state during the transition. In order to eliminate the influence of the reminiscent spin texture on the topological number, the simulation is performed on a system with a ferromagnetic state as the initial state, see Fig.9(c). As the system evolves in time, the range of the $m_{z}$ component expands from $[0.95,0.99]$ to $[0,0.99]$, indicating the departure from a ferromagnetic state and the debut of a skyrmionic one. However, the in-plane component fails to follow this trend and is still of ferromagnetic nature: The in-plane component is largely pointing to 


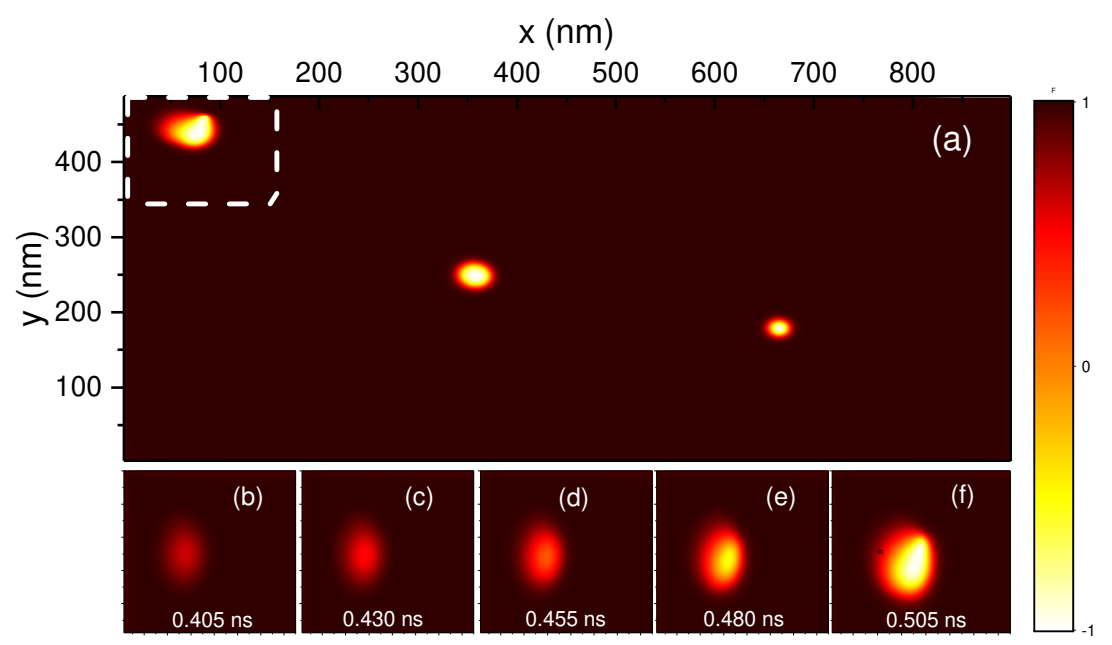

FIG. 8. (a) Snapshot of skyrmions continuously generated by spin current. The current density is $7.2 \times 10^{10} \mathrm{~A} \mathrm{~cm} \mathrm{~cm}^{-2}$. (b)-(f) the temporal evolution of the transition from a droplet to a skyrmion in the rectangle region indicated by the white dashed line. The others parameters are the same as it in Fig. 7.
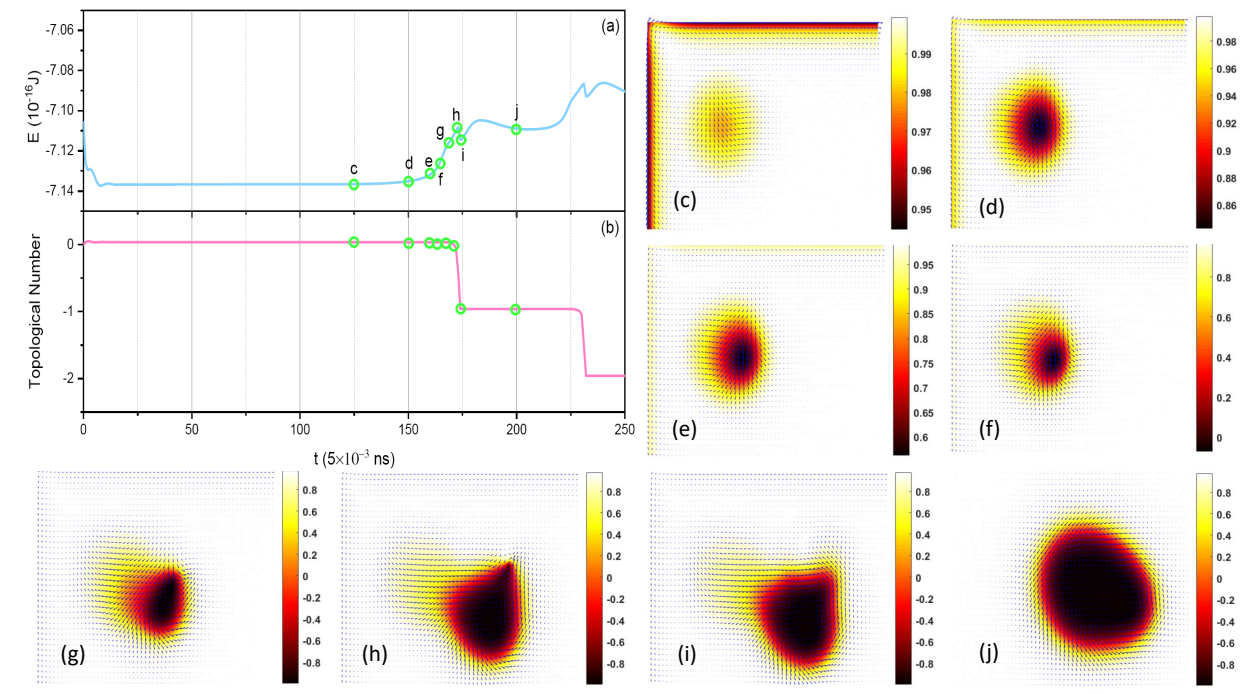

FIG. 9. Time evolution of the magnetization profile during the transition from an unstable ferromagnetic state to a skyrmion. (a) Total energy and (b) topological number versus time. (c)-(j) Magnetization profiles that correspond to points c-j marked by empty green circles in panel (a). Component $m_{z}$ is in contour plot and the direction of in-plane components $\left(m_{x}, m_{y}\right)$ is represented by arrow. 
one direction, as Figs.9(d)-(f) show. This state is what a magnetic droplet, or droplet state, refers to. As the transition progresses further, the magnetization profile transforms into a tear drop shape. The range of $m_{z}$ may have reached $[-1,1]$ but the in-plane component at the top of drop is distorted and it is topologically similar to a curved domain wall, leading to a vanishing topological charge. Only when the droplet has evolved into a skyrmion, the topological charge becomes an integer, which is consistent with the step-like topological charge in Fig.9(b).

\section{SKYRMION MEMORY DEVICE}

(a)

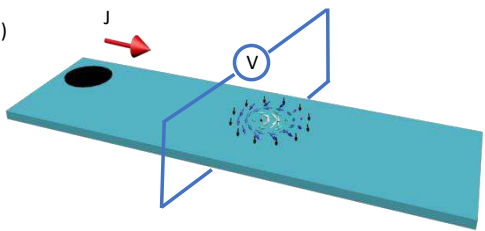

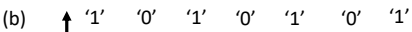

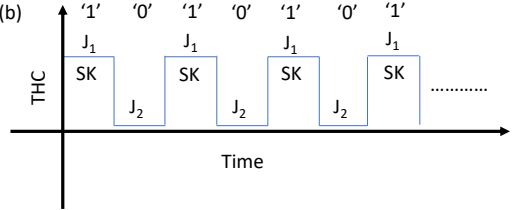

ductivity, we expect a higher value in the measurement. This can be mapped into the '1' state of a binary bit. Absence of skyrmions from the region results in a lower value, which can be regarded as '0' state. As time lapses, we expect an alternative reading the ' 0 ' and ' 1 ' states. Meanwhile, in order to avoid the error of generating ' 0 ' state due to the pinning effect, we propose that 1 ) reduce the diameter of pinning region, increase the current density and extend the duration to capture the skyrmions. 2) after creating skyrmions in the pinning region, transport the skyrmion to a chain of circular defective regions with lower pinning to store skyrmion. 3) it is perhaps beneficial to increase the number of parallel tracks to count for 1 and thus averaging out errors.

\section{CONCLUSIONS}

We have shown that, inhomogeneous perpendicular magnetic anisotropy, when properly designed and patterned on a disk-shaped FeGe based multilayer nanostructure, can mediate the creation and stabilization of skyrmions in zero magnetic field. Upon being embedded into a ferromagnetic conductor, this multilayer nanostructure can functions as a source of skyrmions: skyrmions nucleated in the structure can be driven out continuously and moved along the conductor by a spin polarized current that is above a threshold value. Such a robust skyrmion source can work as a battery to power the next-generation spintronic devices.

\section{AUTHOR CONTRIBUTIONS}

FIG. 10. Schematics of the realization of a binary memory device based on skyrmion. The black circle region stands for a lower PMA compared with the cyan region. The varied current leads to a skyrmion or a uniform state. A switching between $J_{1}$ and $J_{2}$ can tune the creation of skyrmion. When a skyrmion or a nonskyrmionic state moves to the detected region, a different output of transverse voltage would be measured in (b).

Local magnetization switching and nucleation of skyrmion have been observed in experiment ${ }^{43}$. When the initial state is skyrmionic, it is dragged out of the circular pinning region by the spin torque; whereas a random (activated by laser) or a ferromagnetic initial state will first evolve into a skyrmionic state and then move away from the defective region. A ferromag netic initial state, though, requires a higher current density, owing to the higher energy of ferromagnetic state compared to others.

We propose a conceptual device to detect the transverse voltage induced by skyrmion. Consider a two-dimensional device shown in Fig.10(a). It consists of a defective region - as in Fig. 1(a) to generate skyrmions - embedded into a rectangular ferromagnetic nanostrip that has a homogeneous perpendicular anisotropy. Through defective area, skyrmions are generated continuously and driven along the nanostrip by a spin current. When a skyrmion moves across the region where a transverse voltage is measured due to topological Hall con-
H. L. and Z. C supervised the project, H. L. and X. H. peranalyses the data and wrote the paper. All authors commented on the manuscript.

\section{ACKNOWLEDGMENTS}

X. Hao and H. Li acknowledge the support from Henan university (Grant No. CJ3050A0240050) and National Natural Science Foundation of China (Grants No. 11804078). A. Manchon acknowledges support from the Excellence Initiative of Aix-Marseille Université - A*Midex, a French "Investissements d'Avenir" program. F. Zhuo acknowledges the support from King Abdullah University of Science and Technology (KAUST). Z.X. Cheng acknowledges the support from ARC(DP190100150). H. Li acknowledges the support from KAUST at the beginning of his career.

\section{DATA AVAILABILITY}

The data that support the findings of this study are available formed the theoretical calculations, H. L., F. Z., A. M and Z. C from the corresponding author upon reasonable request. 


\section{REFERENCES}

${ }^{1}$ A. N. Bogdanov and U. K. Rößler, Phys. Rev. Lett. 87, 037203 (2001). ${ }^{2}$ N. Nagaosa and Y. Tokura, Nat. Nano. 8, 899 (2013).

${ }^{3}$ A. Fert, N. Reyren and V. Cros, Nat. Rev. Mat. 2, 17031 (2017).

${ }^{4}$ S. Mühlbauer, B. Binz, F. Jonietz, C. Pfleiderer, A. Rosch, A. Neubauer, R. Georgii and P. Böni, Science 323, 915 (2009).

${ }^{5}$ S. Heinze, K. von Bergmann, M. Menzel, J. Brede, A. Kubetzka, R. Wiesendanger, G. Bihlmayer, S. Blugel, Nat. Phys. 7, 713 (2011). ${ }^{6}$ S. Seki, X. Z. Yu, S. Ishiwata, and Y. Tokura, Science 336, 198 (2012).

${ }^{7}$ A. K. Nayak, V. Kumar, T. Ma, P. Werner, E. Pippel, R. Sahoo, F. Damay, U. K. Rößler, C. Felser, and S. S. P. Parkin, Nature 548, 561 (2017).

${ }^{8}$ L. Wang, Q. Feng, Y. Kim, R. Kim, K. Lee, S. D. Pollard, Y. J. Shin, H. Zhou, W. Peng, D. Lee, W. Meng, H. Yang, J. Hoon, M. Kim, Q. Lu and T. Noh, Nat. Mater. 17, 1087 (2018).

${ }^{9}$ B. Ding, Z. Li, G. Xu, H. Li, Z. Hou, E. Liu, X. K. Xi, F. Xu, Y. Yao, and W. Wang, Nano Lett. 20, 868 (2020).

${ }^{10}$ M. Yang, Q. Li, R. V. Chopdekar, R. Dhall, J. Turner, J. D. Carlström, C. Ophus, C. Klewe, P. Shafer, A. T. N'Diaye, J. W. Choi, G. Chen, Y. Z. Wu6, C. Hwang, F. Wang and Z. Q. Qiu, Sci. Adv. 6, 5157 (2020).

${ }^{11}$ P. Milde, D. Köhler, J. Seidel, L. M. Eng, A. Bauer, A. Chacon, J. Kindervater, S. Mühlbauer, C. Pfleiderer, S. Buhrandt, C. Schütte and A. Rosch, Science 340, 1076 (2013).

${ }^{12}$ N. Romming, C. Hanneken, M. Menze, J. E. Bickel, B. Wolter, K. v. Bergmann, A. Kubetzka and R. Wiesendanger, Science 341, 636 (2013).

${ }^{13}$ P. Hsu, A. Kubetzka, A. Finco, N. Romming, K. Bergmann and R. Wiesendanger, Nat. Nano. 12, 123 (2017)

${ }^{14}$ G. Berruto, I. Madan, Y. Murooka, G. M. Vanacore, E. Pomarico, J. Rajeswari, R. Lamb, P. Huang, A. J. Kruchkov, Y. Togawa, T. LaGrange, D. McGrouther, H. M. Rønnow, and F. Carbone, Phys. Rev. Lett. 120, 11720 (2018).

${ }^{5}$ T. Yokouchi, S. Sugimoto, B. Rana, S. Seki, N. Ogawa, S. Kasai, Y. Otani, Nat. Nano. 15, 361 (2020)

${ }^{16}$ K. Shibata, J. Iwasaki, N. Kanazawa, S. Aizawa, T. Tanigaki, M. Shirai, T. Nakajima, M. Kubota, M. Kawasaki, H. S. Park, D. Shindo, N. Nagaosa, Y Tokura, Nat. Nano. 10, 589 (2015).

${ }^{17}$ X. Yu, M. Mostovoy, Y. Tokunaga, W. Zhang, K. Kimoto, Y. Matsui, Y. Kaneko, N. Nagaosa, Y. Tokura, Pro. Nat. Aca. Sci. 109, 8856 (2012).

${ }^{18}$ X. Z. Yu, W. Koshibae, Y. Tokunaga, K. Shibata, Y. Taguchi, N. Nagaosa, and Y. Tokura, Nature 564, 95 (2018).

${ }^{19}$ Y. A. Kharkov, O. P. Sushkov, and M. Mostovoy, Phys. Rev. Lett. 119 207201 (2017).

${ }^{20}$ N. Gao, S. Je, M. Im, J. Choi, M. Yang, Q. Li, T.Y. Wang, S. Lee, H. S Han, K. S. Lee, W. Chao, C. Hwang, J. Li, Z.Q. Qiu, Nat. Commun 10 5603 (2019).

${ }^{21}$ W. Sun, W. Wang, H. Li, G. Zhang, D. Chen, J. Wang and Z. Cheng, Nat Commun 11, 5930 (2020).

${ }^{22}$ F. Zheng, F. Rybakov, A. Borisov, D. Song, S. Wang, Z. Li, H. Du, N. Kiselev, J. Caron, A. Kovacs, M. Tian, Y. Zhang, S. Blugel and R. DuninBorkowski, Nat. Nano., 12, 451 (2018).

${ }^{23}$ S. Gao, H.D. Rosales, F. Albarracín, V. Tsurkan, G. Kaur, T. Fennell, P. Steffens, M. Boehm, P. Čermák, A. Schneidewind, E. Ressouche, D. C. Cabra, C. Rüegg and O Zaharko, Nature 586, 37 (2020).

${ }^{24}$ F. Jonietz, S. Mühlbauer, C. Pfleiderer, A. Neubauer, W. Münzer, A. Bauer, T. Adams, R. Georgii, P. Böni,R. Duine, K. Everschor, M. Garst, A. Rosch, Science 330, 1648 (2010).

${ }^{25}$ L. Kong and J. Zang, Phys. Rev. Lett. 111, 067203 (2013).

${ }^{26}$ A. Fert, V. Cros and J. Sampaio, Nat. Nano. 8, 152 (2013).

${ }^{27}$ X. Zhang, M. Ezawa, and Y. Zhou, Sci. Rep. 5, 9400 (2015).

${ }^{28}$ S. Luo, M. Song, X. Li, Y. Zhang, J. Hong, X. Yang, X. Zou, N. Xu, and L. You, Nano Lett. 18, 1180 (2018)

${ }^{29}$ D. Pinna, F. Abreu Araujo, J. V. Kim, V. Cros, D. Querlioz, P. Bessiere, J. Droulez, and J. Grollier, Phys. Rev. App. 9, 064018 (2018).

${ }^{30}$ K. Song, J. Jeong, B. Pan, X. Zhang, J. Xia, S. Cha, T. Park, K. Kim, S Finizio, J. Raabe, J. Chang, Y. Zhou, W. Zhao, W. Kang, H. Ju and S. Woo, Nat. Electron 3, $148(2020)$

${ }^{31}$ W. Legrand, D. Maccariello, N. Reyren, K. Garcia, C. Moutafis, C. MoreauLuchaire, S. Collin, K. Bouzehouane, V. Cros, and A. Fert, Nano Lett 17 2703 (2017).
${ }^{32}$ S. Woo, K. M. Song, H.-S. Han, M.-S. Jung, M.-Y. Im, K.-S. Lee, K. S. Song, P. Fischer, J.-I. Hong, J. W. Choi, B.-C. Min, H. C. Koo, and J. Chang, Nat. Commun. 8, 15573 (2017)

${ }^{33}$ H. Y. Yuan and X. R. Wang, Sci. Rep. 6, 22638 (2016).

${ }^{34}$ W. Koshibae, N. Nagaosa, https://doi.org/10.1038/s41598-018-24693-5Sci. Rep. 8, 6328 (2018)

${ }^{35}$ J. Sampaio, V. Cros, S. Rohart, A. Thiaville and A. Fert, Nat. Nano. 8, 839 (2013).

${ }^{36}$ F. Zheng, H. Li, S. Wang, D. Song, C. Jin, W. Wei, A. Kovács, J. Zang, M. Tian, Y. Zhang, H. Du, and R. E. Dunin-Borkowski, Phys. Rev. Lett. 119, 197205 (2017).

${ }^{37}$ D. A. Gilbert, B. B. Maranville, A. L. Balk, B. J. Kirby, P. Fischer, D. T. Pierce, J. Unguris, J. A. Borchers and K. Liu, Nat. Commun. 6, 8462 (2015)

${ }^{38}$ J. Li, A. Tan, K. Moon, A. Doran, M. Marcus, A. Young, E. Arenholz, S. Ma, R. Yang, C. Hwang and Z. Qiu, Nat. Commun. 5, 4704 (2014).

${ }^{39}$ Z. Wang, Z. Li, R. Wang, B. Liu, H. Meng, Y. Cao, and P. Yan, Appl. Phys. Lett. 117, $222406(2020)$

${ }^{40} \mathrm{X}$. Zhao, C. Jin, C. Wang, H. Du, J. Zang, M. Tian, R. Che, and Y. Zhang, Proc. Natl. Acad. Sci. U.S.A. 113, 4918 (2016)

${ }^{41}$ B. Dupé, G. Bihlmayer, M. Böttcher, S. Bügel and S. Heinze, Nat. Commun. 7, 11779 (2016)

${ }^{42}$ X. Yu, N. Kanazawa, Y. Onose, K. Kimoto, W. Zhang, S. Ishiwata, Y. Matsui and Y. Tokura, Nat. Mater. 10, 106 (2011).

${ }^{43}$ F. Büttner, I. Lemesh, M. Schneider, B. Pfau, C. Günther, P. Hessing, J. Geilhufe, L. Caretta, D. Engel, B. Krüger, J. Viefhaus, S. Eisebitt and G. Beach, Nat. Nano. 12, 1040 (2017).

${ }^{44}$ K. Everschor-Sitte, M. Sitte, T. Valet, A. Abanov and J. Sinova, New J. Phys. 19092001 (2017).

${ }^{45}$ I. Fernandes, J. Bouaziz, S. Blügel, S. Lounis, Nat. Commun. 9, 4395 (2018).

${ }^{46}$ K. Fallon,S. Hughes,K. Zeissler,W. Legrand, F. Ajejas, D. Maccariello, S. McFadzean, W. Smith, D. McGrouther, S. Collin, N. Reyren, V. Cros, C. H. Marrows, S. McVitie, Small 16, 1907450 (2020).

${ }^{47}$ J. Iwasaki, M. Mochizuki and N. Nagaosa, Nat. Nano. 8, 742 (2013).

${ }^{48}$ W. Jiang, P. Upadhyaya, W. Zhang, G. Yu, M. Benjamin Jungfleisch, F. Fradin, J. Pearson, Y. Tserkovnyak, K. Wang, O. Heinonen, S. Velthuis, A. Hoffmann, Science, 349, 283 (2015).

${ }^{49}$ Z. Luo, T. Dao, A. Hrabec, J. Vijayakumar, A. Kleibert, M. Baumgartner, E. Kirk, J. Cui, T. Savchenko, G. Krishnaswamy, L. J. Heyderman, P. Gambardella, Science, 363, 1435 (2019).

${ }^{50}$ C. Song, C. Jin, J. Wang, Y. Ma, H. Xia, J. Wang, J. Wang and Q. Liu, Appl. Phys. Express 120830032019

${ }^{51}$ H. Xia, C. Song, C. Jin, J. Wang, J. Wang and Q. Liu, J. MMM 57458 (2018)

${ }^{52}$ Y. Zhou, R. Mansell and S. V. Dijken, Sci. Rep. 9, 6525 (2019).

${ }^{53}$ C. Ang, W. Gan and W. Lew, New J. Phys. 210430062019

${ }^{54}$ S. X. Huang and C. L. Chien, Phys. Rev. Lett. 108, 267201 (2012).

${ }^{55}$ Nicholas A. Porter, Charles S. Spencer, Rowan C. Temple, Christian J. Kinane, Timothy R. Charlton, Sean Langridge, and Christopher H. Marrows, Phys. Rev. B 92, 144402 (2015).

${ }^{56}$ Z. Xie, Y. Li, Z. Gong, X. Yang, Y. Li, R. Sun, N. Li, Y. Sun, J. Zhao, Z. Cheng, and W. He, ACS Appl. Mater. Interfaces 12, 46908 (2020).

${ }^{57}$ U. Rucker, S. Demokritov, R. Arons and P. Grunberga, JMMM, 156, 269 (1996)

${ }^{58}$ T. Maruyama, Y. Shiota, T. Nozaki, K. Ohta, N. Toda, M. Mizuguchi, A. A Tulapurkar, T. Shinjo, M. Shiraishi, S. Mizukami, Y. Ando and Y. Suzuki, Nat. Nano. 4, 158 (2009).

${ }^{59}$ T. Nozaki, A. Kozioł-Rachwał, M. Tsujikawa, Y. Shiota, X. Xu, T. Ohkubo, T. Tsukahara, S. Miwa, M. Suzuki, S. Tamaru, H. Kubota, A. Fukushima, K. Hono, M. Shirai, Y. Suzuki and S. Yuasa, NPG Asia Mater, 9, e451 (2017)

${ }^{60}$ S. Zhao, Z. Zhou, C. Li, B. Peng, Z. Hu and M. Liu, ACS Nano 7, 7167

${ }^{61}$ W. F. Brown, Micromagnetics, Interscience Publishers, New York, NY,
$\quad$. 1963.

${ }^{62}$ A. Vansteenkiste, J. Leliaert, M. Dvornik, M. Helsen, F. Garcia-Sanchez and B. Waeyenberge, AIP Advances 4, 107133 (2014).

${ }^{63}$ R.D. McMichael, M.J. Donahue, D.G. Porter, and Jason Eicke, J. App. Phys. 85, 5816 (1999). 
${ }^{64}$ C. Shir, IEEE Transactions on Magnetics 17, 2401 (1981). ${ }^{6}$ L. Liu, C. Pai, Y. Li, H. W. Tseng, D. C. Ralph, R. A. Buhrman, Science 336, 555 (2012)

${ }^{66} \mathrm{C}$. Moreau-Luchaire, C. Moutafis, N. Reyren, J. Sampaio, C. Vaz, N. Horne, K. Bouzehouane, K. Garcia, C. Deranlot, P. Warnicke, P. Wohlhüter, J.-M George, M. Weigand, J. Raabe, V. Cros and A. Fert, Nat. Nano. 11, 444 (2016)

${ }^{67}$ A. N. Bogdanov and D. A. Yablonskii, Zh. Eksp. Teor. Fiz., 95, 178 (1989).

${ }^{68}$ A. Bogdanov and A. Hubert, J. MMM, 195, 182 (1999).
${ }^{69}$ R. Pepper, M. Beg, D. Cortes-Ortuno, T. Kluyver, M. Bisotti, R. Carey, M. Vousden, M. Albert, W. Wang, O. Hovorka, and H. Fangohr, J. App. Phys. 123, 093903 (2018).

${ }^{70}$ S. Woo, K. Litzius, B. Krüger, M. Im, L. Caretta, K. Richter, M. Mann, A. Krone, R. Reeve, M. Weigand, P. Agrawal, I. Lemesh, M. Mawass, P. Fischer, M. Kläui and G. Beach, Nat. Mat. 15, 501 (2016).

${ }^{71}$ S. Rohart and A. Thiaville, Phys. Rev. B 88, 184422 (2013).

${ }^{72}$ J. Iwasaki, M. Mochizuki, and N. Nagaosa, Nat. Commun. 4, 1463 (2013). ${ }^{73}$ Jing Xia, Xichao Zhang, Motohiko Ezawa, Zhipeng Hou, Wenhong Wang, Xiaoxi Liu, and Yan Zhou, Phys. Rev. App. 11, 044046 (2019). 


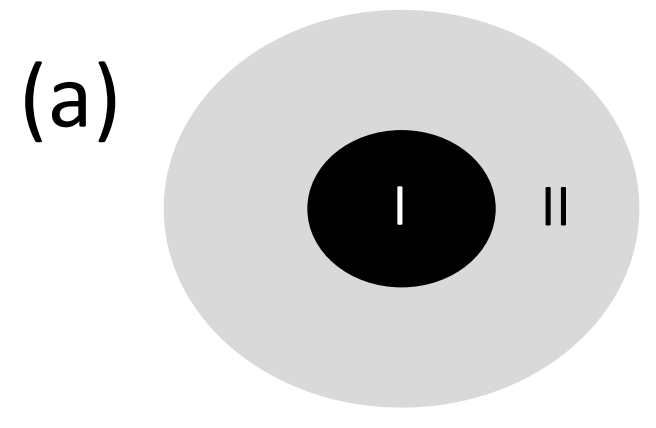

Top view

(c)

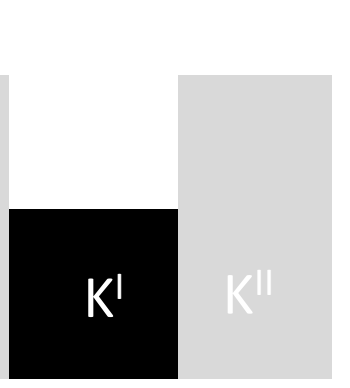

(b)

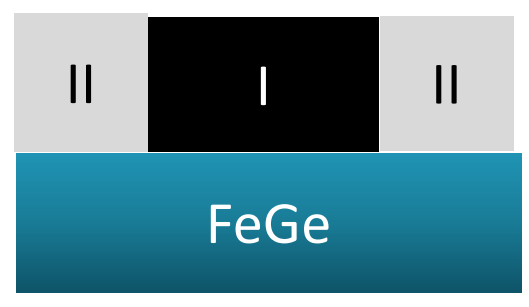

Side view

(d) 

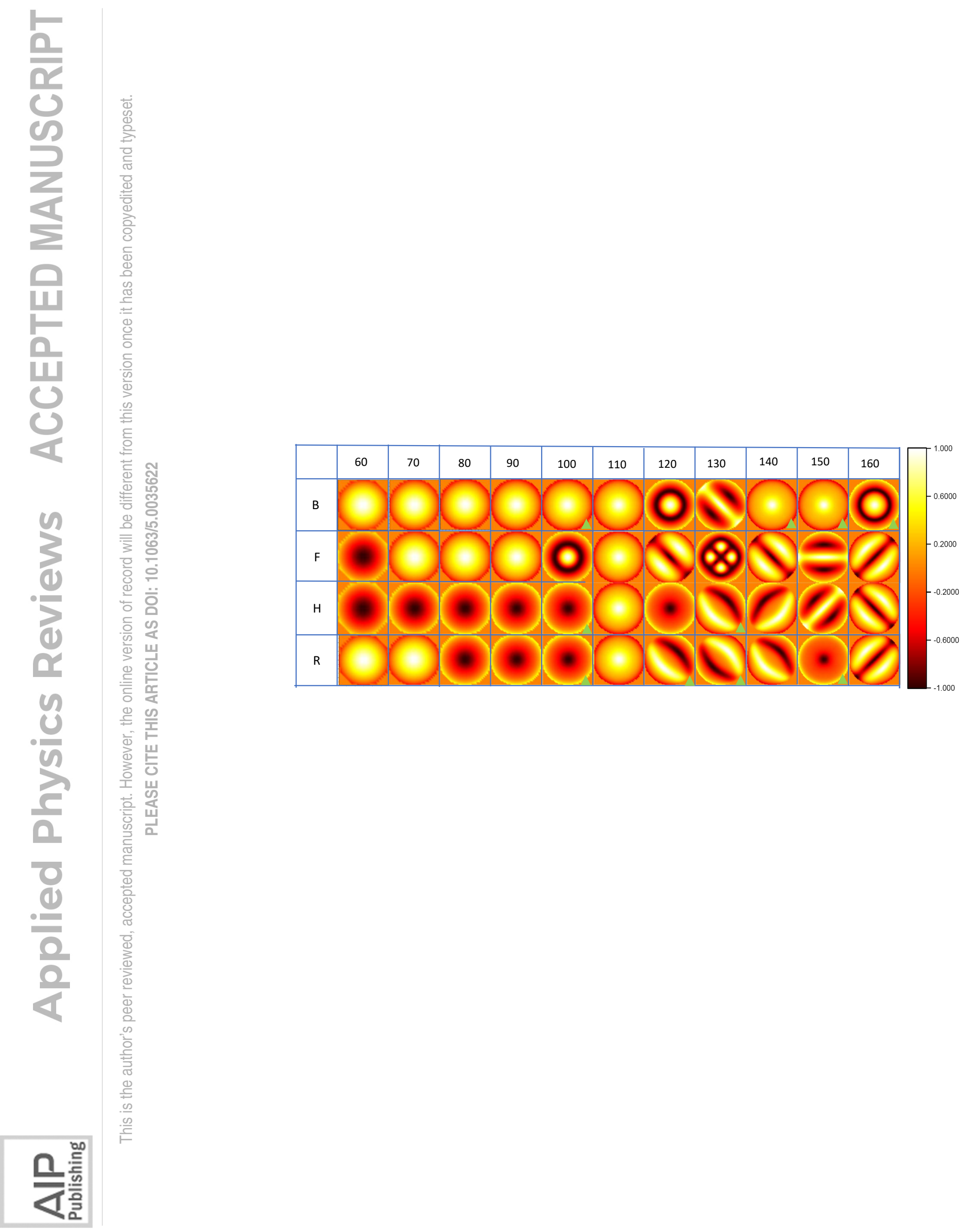

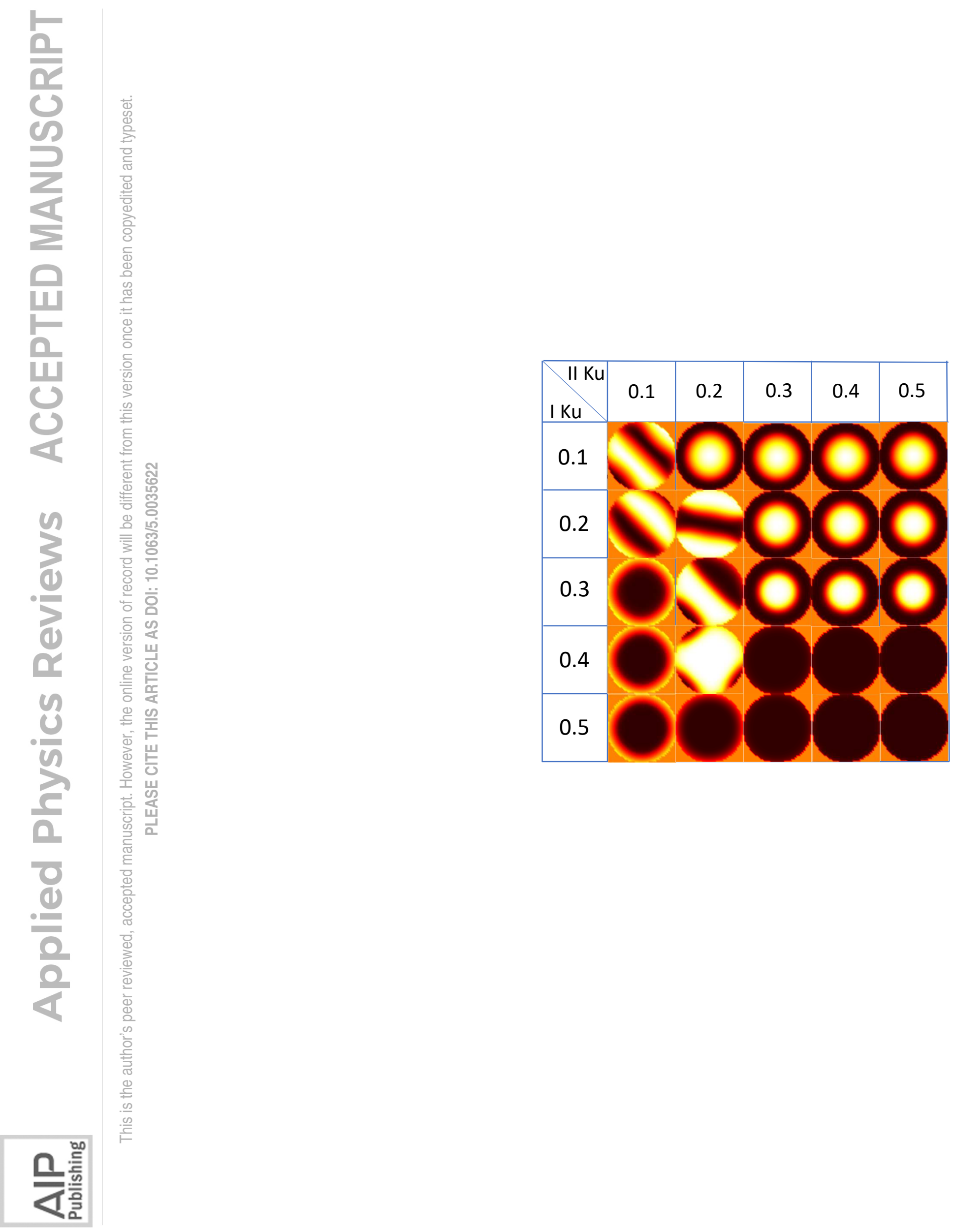

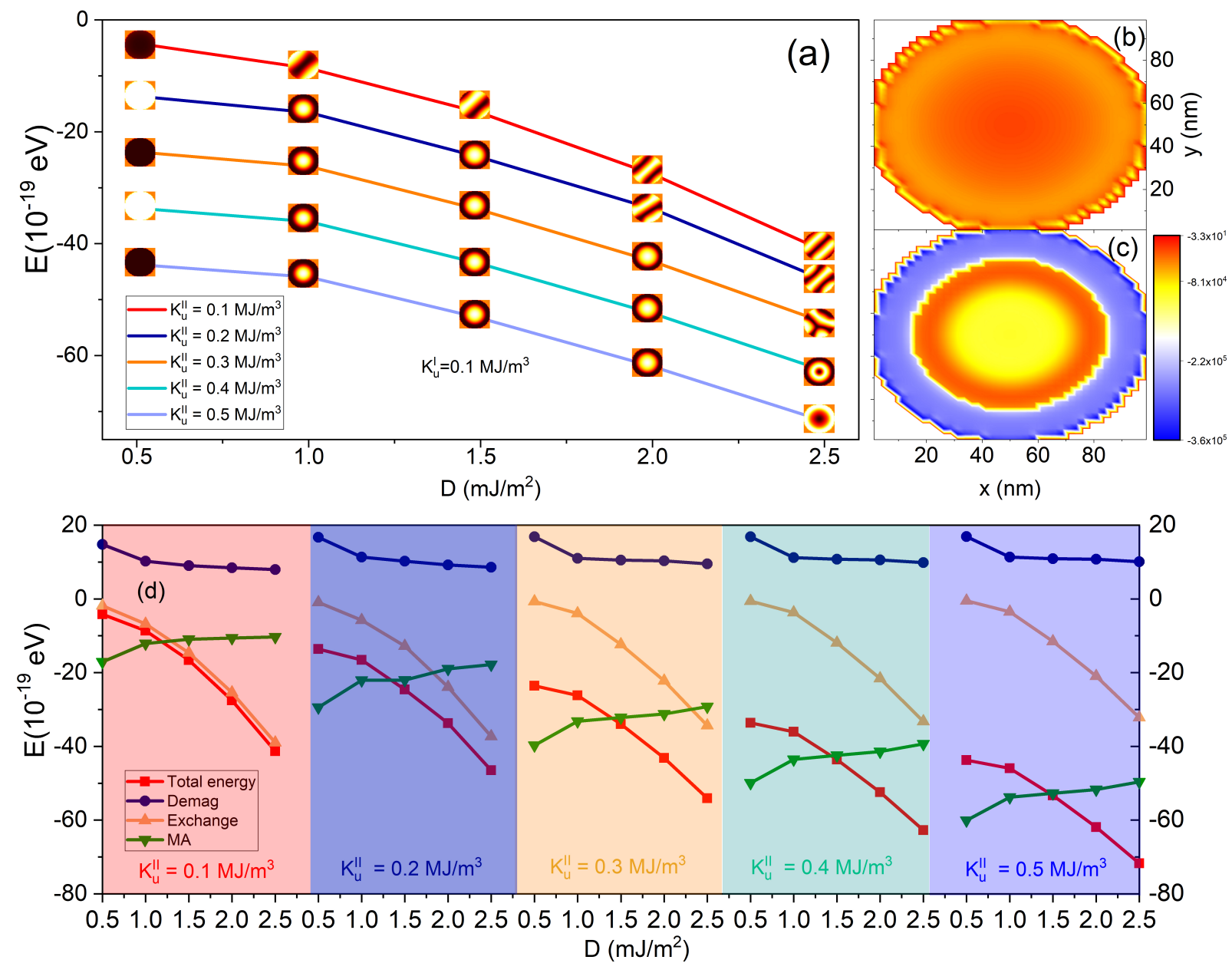

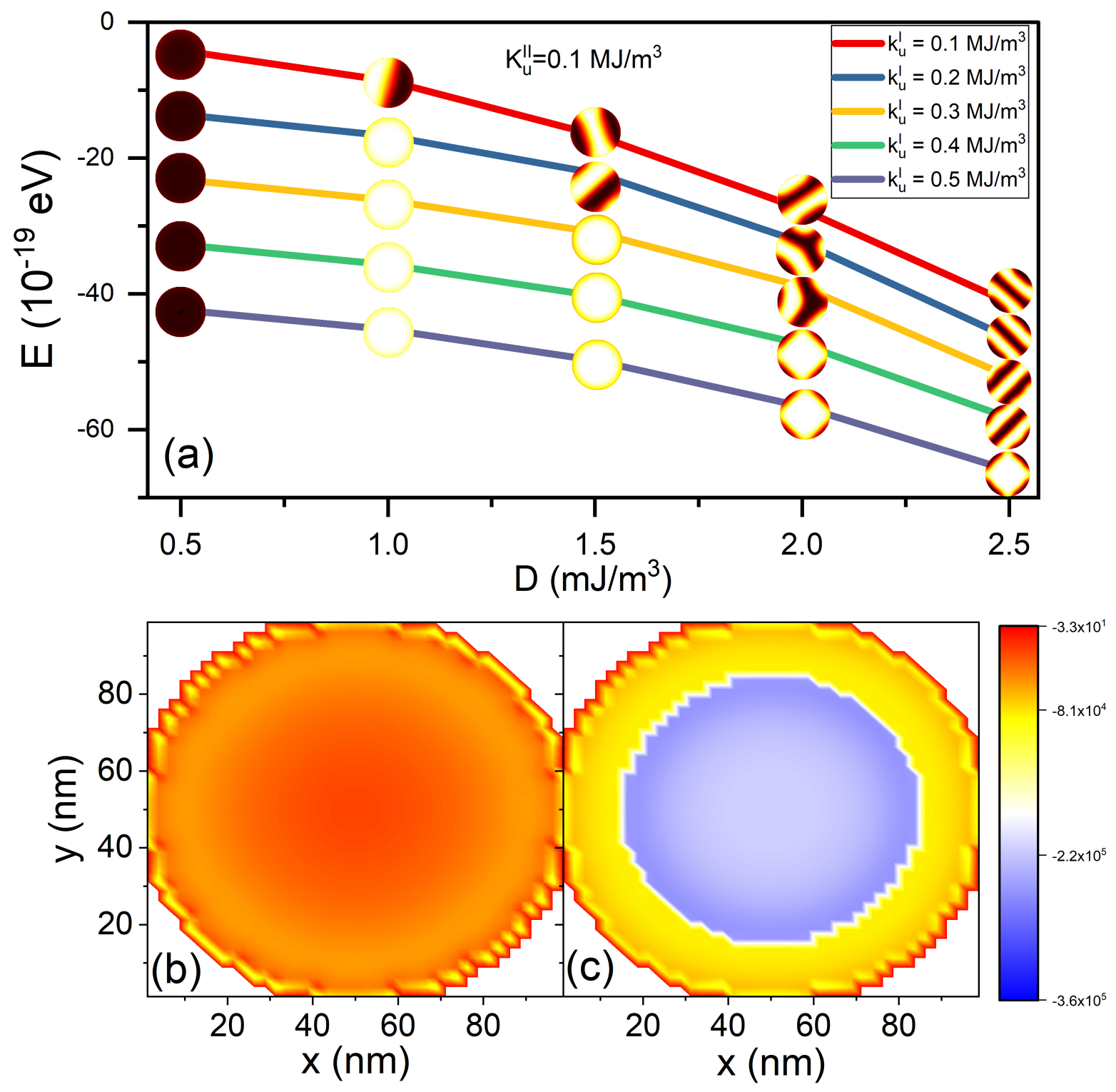

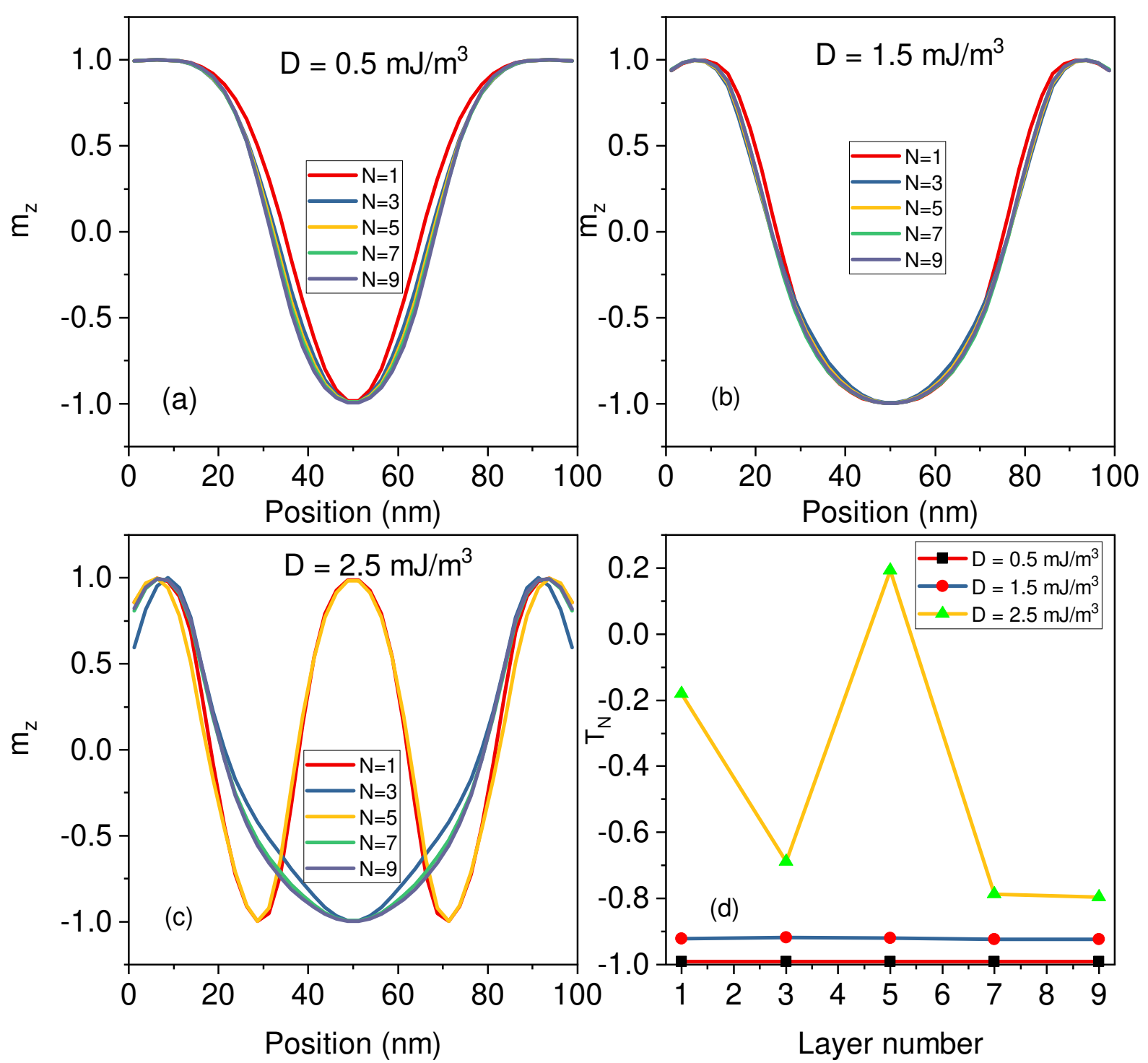

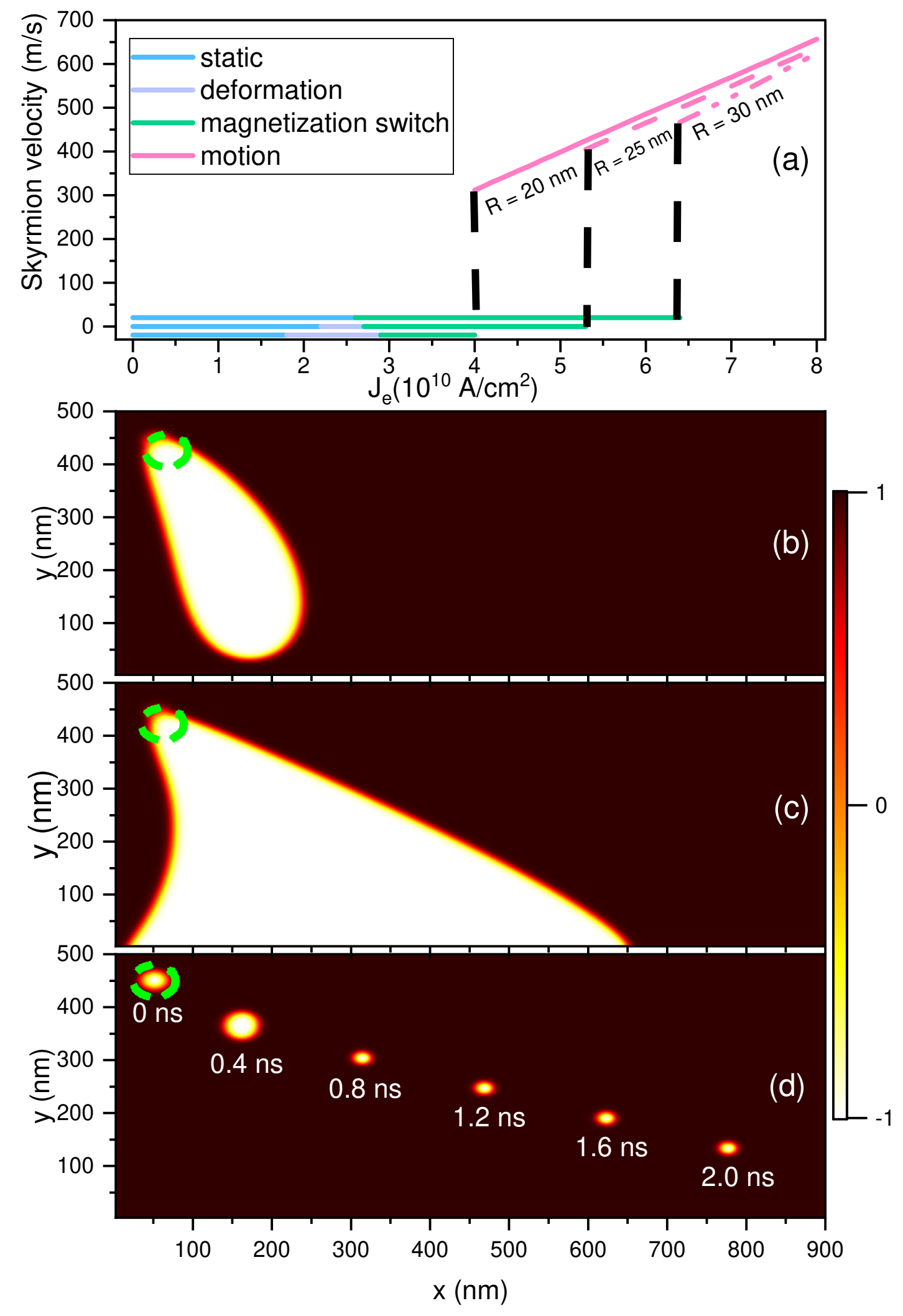


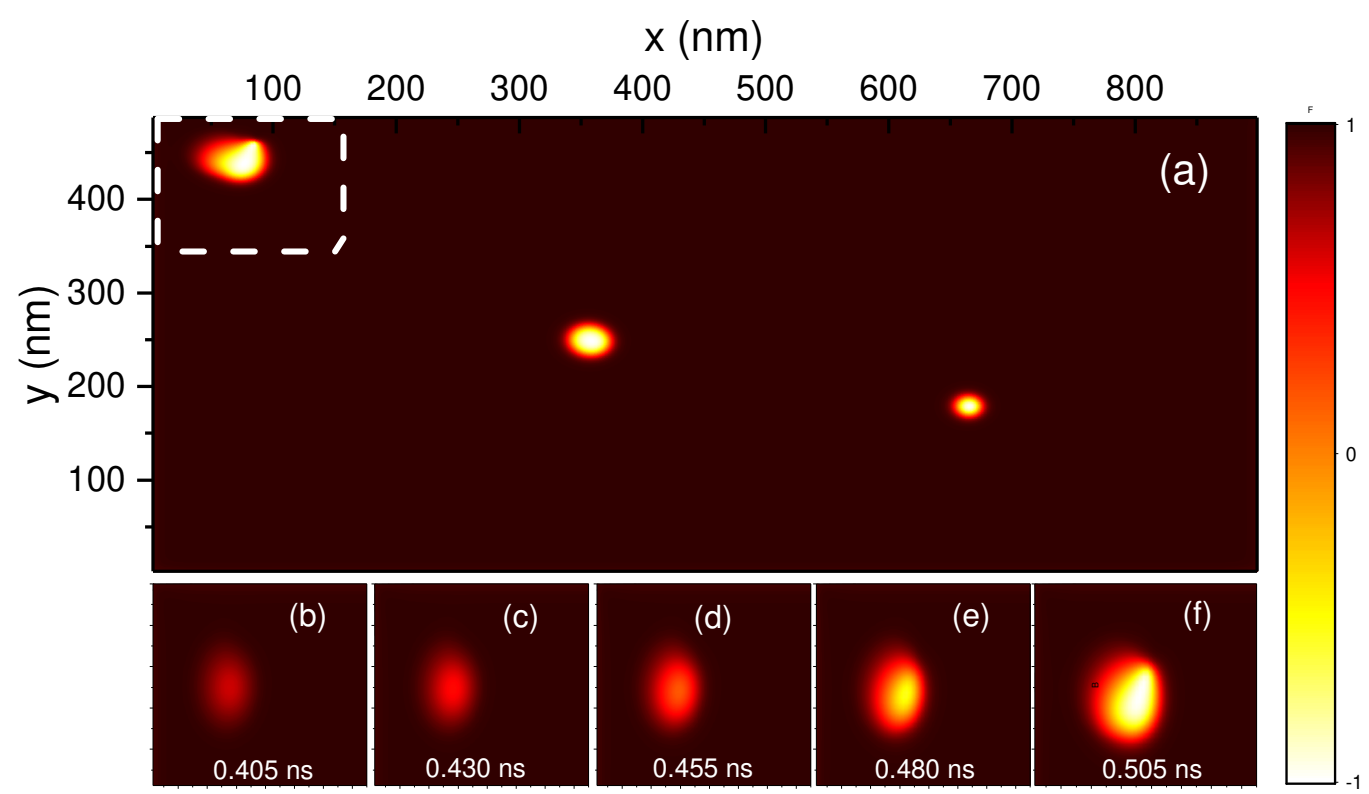




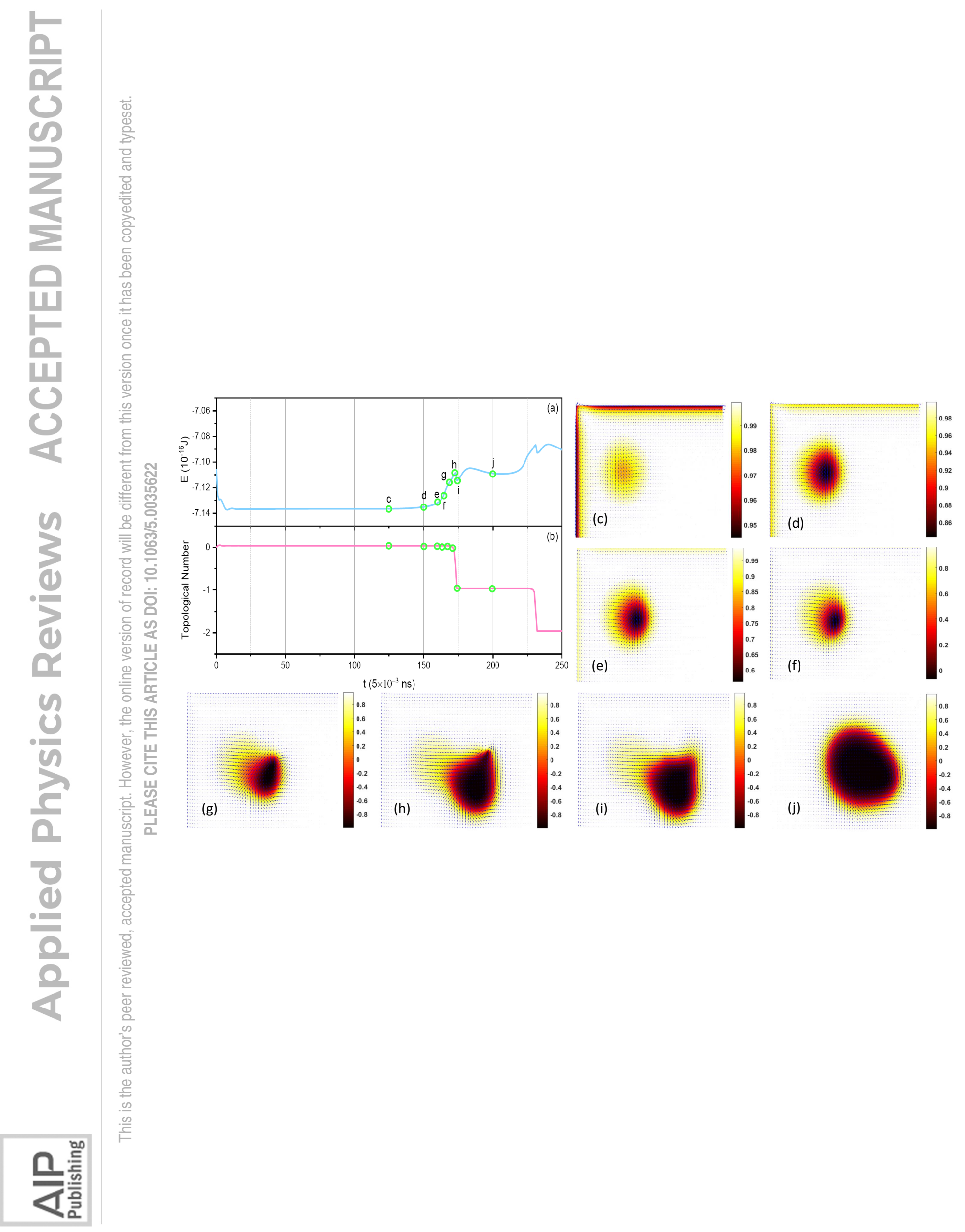




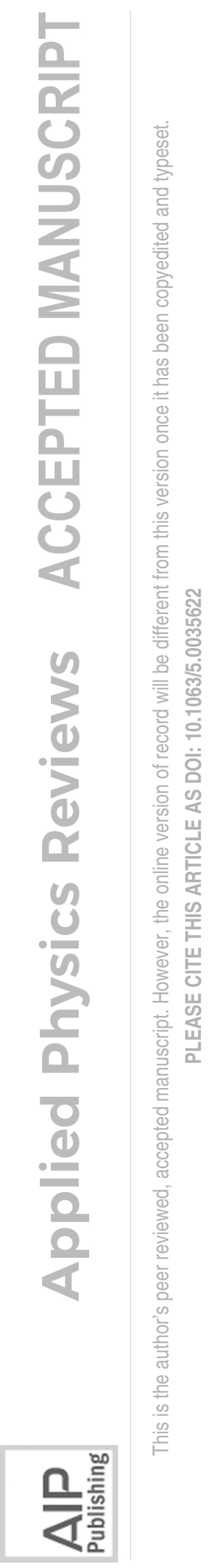

(a)
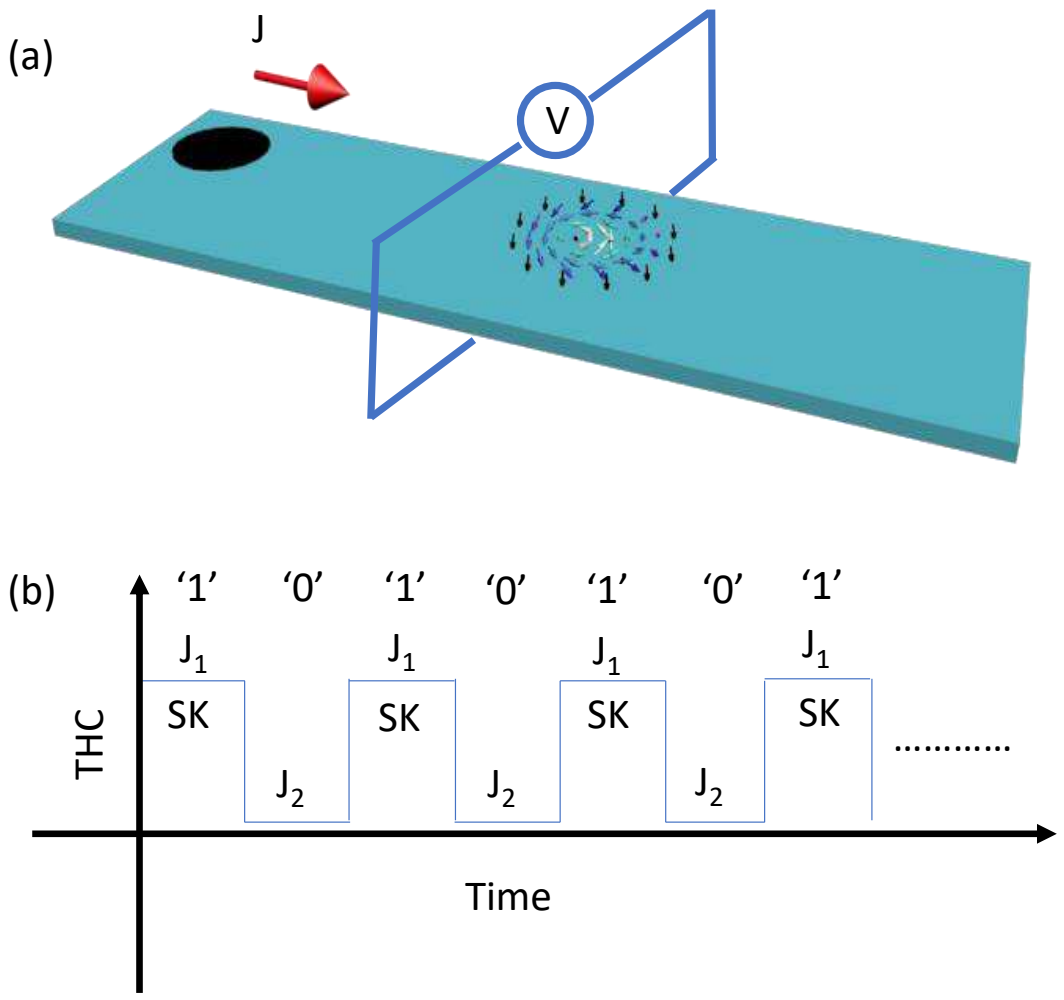